\title{
Quantum regression theorem and non-Markovianity of quantum dynamics
}

\author{
Giacomo Guarnieri, ${ }^{1,2}$ Andrea Smirne, ${ }^{3,4}$ and Bassano Vacchini ${ }^{1,2}$ \\ ${ }^{1}$ Dipartimento di Fisica, Università degli Studi di Milano, Via Celoria 16, 20133 Milan, Italy \\ ${ }^{2}$ Istituto Nazionale di Fisica Nucleare, Sezione di Milano, Via Celoria 16, 20133 Milan, Italy \\ ${ }^{3}$ Dipartimento di Fisica, Università degli Studi di Trieste, Strada Costiera 11, 34151 Trieste, Italy \\ ${ }^{4}$ Istituto Nazionale di Fisica Nucleare, Sezione di Trieste, Via Valerio 2, 34127 Trieste, Italy
}

(Received 12 June 2014; published 15 August 2014)

\begin{abstract}
We explore the connection between two recently introduced notions of non-Markovian quantum dynamics and the validity of the so-called quantum regression theorem. While non-Markovianity of a quantum dynamics has been defined looking at the behavior in time of the statistical operator, which determines the evolution of mean values, the quantum regression theorem makes statements about the behavior of system correlation functions of order two and higher. The comparison relies on an estimate of the validity of the quantum regression hypothesis, which can be obtained exactly evaluating two-point correlation functions. To this aim we consider a qubit undergoing dephasing due to interaction with a bosonic bath, comparing the exact evaluation of the non-Markovianity measures with the violation of the quantum regression theorem for a class of spectral densities. We further study a photonic dephasing model, recently exploited for the experimental measurement of nonMarkovianity. It appears that while a non-Markovian dynamics according to either definition brings with itself violation of the regression hypothesis, even Markovian dynamics can lead to a failure of the regression relation.
\end{abstract}

DOI: 10.1103/PhysRevA.90.022110

PACS number(s): 03.65.Yz, 42.50.Lc, 03.67.-a

\section{INTRODUCTION}

In recent times there has been a revival in the study of the characterization of non-Markovianity for an open quantum system dynamics. While the subject was naturally born together with the introduction of the first milestones in the description of the time evolution of a quantum system interacting with an environment $[1,2]$, the difficulty inherent in the treatment led to very few general results, and the very definition of a convenient notion of Markovian open quantum dynamics was not agreed upon. The focus initially was on finding the closest quantum counterpart of the classical notion of Markovianity for a stochastic process, so that reference was made to correlation functions of all order for the process. Recent work was rather focused on proposals of a notion of Markovian quantum dynamics based on an analysis of the behavior of the statistical operator describing the system of interest only, thus concentrating on features of the dynamical evolution map, which only determines mean values. Different properties of the time evolution map have been considered in this respect [3-12]. In particular two viewpoints [4,6] appear to have captured important aspects in the characterization of a dynamics which can be termed non-Markovian in the sense that it relates to memory effects.

The aim of our work is to analyze the relationship between these approaches and the validity of the so-called quantum regression theorem [13,14], according to which the behavior in time of higher order correlation functions can be predicted building on the knowledge of the dynamics of the mean values for a generic observable. The analysis can be performed introducing a suitable quantifier for the violation of the quantum regression hypothesis, which in turn requires knowledge of the exact two-time correlation functions. We therefore consider a two-level system coupled to a bosonic bath through a decoherence interaction, exactly estimating for a general class of spectral densities the predictions of different criteria for non-Markovianity of a dynamics and the violation of the regression theorem. We further apply this analysis to a dephasing model, whose realization has been recently exploited to experimentally observe quantum nonMarkovianity [15]. In both cases we show that the quantum regression theorem can be violated even in the presence of a quantum dynamics which, according to either criteria, is considered Markovian.

The paper is organized as follows. In Sec. II we recall two recently introduced notions of Markovianity for a quantum dynamics and the associated measures, while in Sec. III we address the formulation of the quantum regression theorem and introduce a simple estimator for its violation. We apply this formalism to the pure dephasing spin boson model in Sec. IV discussing the relationship between the two approaches, and extend the analysis to a photonic dephasing model in Sec. V. We finally comment on our results in Sec. VI.

\section{NON-MARKOVIANITY DEFINITIONS AND MEASURES}

Let us start by briefly recalling the main features of the notion of non-Markovian quantum dynamics which will be exploited in the following analysis. In the classical theory of stochastic processes, the definition of Markov process involves the entire hierarchy of $n$-point joint probability distributions associated with the process. Since such a definition cannot be directly transposed to the quantum realm [16,17], different and nonequivalent notions of quantum Markovianity have been introduced [3-11], along with different measures to quantify the degree of non-Markovianity of a given dynamics (see $[18,19]$ for a very recent comparison). These definitions all convey the idea that the occurrence of memory effects is the proper attribute of non-Markovian dynamics, relying on different properties of the dynamical maps which describe the evolution of the open quantum system. In the absence of initial correlations between the open system and its environment, i.e.,

$$
\rho_{S E}(0)=\rho_{S}(0) \otimes \rho_{E}(0)
$$


with $\rho_{E}(0)$ assumed to be fixed, the evolution of an open quantum system is characterized by a one-parameter family of completely positive and trace preserving (CPT) maps $\{\Lambda(t)\}_{t \geqslant 0}$, such that [13]

$$
\rho_{S}(t)=\Lambda(t) \rho_{S},
$$

where $\rho_{S} \equiv \rho_{S}(0)$ is the state of the open system at the initial time $t_{0}=0$. A relevant class of open quantum system dynamics is provided by the semigroup ones, which are characterized by the composition law

$$
\Lambda(t) \Lambda(s)=\Lambda(t+s) \quad \forall t, s \geqslant 0 .
$$

The generator of a semigroup of CPT maps is fixed by the Gorini-Kossakowski-Sudarshan-Lindblad theorem $[1,20]$, which implies that the dynamics of the system is given by the Lindblad equation

$$
\begin{aligned}
\frac{d}{d t} \rho_{S}(t)= & -i\left[H, \rho_{S}(t)\right] \\
& +\sum_{k} \gamma_{k}\left(L_{k} \rho_{S}(t) L_{k}^{\dagger}-\frac{1}{2}\left\{L_{k}^{\dagger} L_{k}, \rho_{S}(t)\right\}\right)
\end{aligned}
$$

with $\gamma_{k} \geqslant 0$. The semigroups of CPT maps are identified with the Markovian time-homogeneous dynamics according to all the previously mentioned definitions of Markovianity, so that the differences between them actually concern the notion of time-inhomogeneous Markovian dynamics.

In the following, we will take into account two definitions of Markovianity and the corresponding measures of nonMarkovianity. One definition [4] is related with the contractivity of the trace distance under the action of the dynamical maps, while the other [6] relies on a divisibility property of the dynamical maps, which reduces to the semigroup composition law in the time-homogeneous case.

\section{A. Trace-distance measure}

The basic idea behind the definition of non-Markovianity introduced by Breuer, Laine, and Piilo (BLP) [4] is that a change in the distinguishability between the reduced states can be read in terms of an information flow between the open system and the environment. The distinguishability between quantum states is quantified through the trace distance [21], which is the metric on the space of states induced by the trace norm:

$$
D\left(\rho^{1}, \rho^{2}\right)=\frac{1}{2}\left\|\rho^{1}-\rho^{2}\right\|_{1}=\frac{1}{2} \sum_{k}\left|x_{k}\right|,
$$

where the $x_{k}$ are the eigenvalues of the traceless Hermitian operator $\rho^{1}-\rho^{2}$. The trace distance takes values between 0 and 1 and, most importantly, it is a contraction under the action of CPT maps. By investigating the evolution of the trace distance between two states of the open system coupled to the same environment but evolved from different initial conditions,

$$
D\left(t, \rho_{S}^{1,2}\right) \equiv D\left(\rho_{S}^{1}(t), \rho_{S}^{2}(t)\right), \quad \rho_{S}^{k}(t)=\Lambda(t) \rho_{S}^{k},
$$

one can thus describe the exchange of information between the open system and the environment. A decrease of the trace distance $D\left(t, \rho_{S}^{1,2}\right)$ means a lower ability to discriminate between the two initial conditions $\rho_{S}^{1}$ and $\rho_{S}^{2}$, which can be expressed by saying that some information has flown out of the open system. On the same ground, an increase of the trace distance can be ascribed to a backflow of information to the open system and then represents a memory effect in its evolution. Non-Markovian quantum dynamics can be thus defined as those dynamics which present a nonmonotonic behavior of the trace distance, i.e., such that there are time intervals $\Omega_{+}$in which

$$
\sigma\left(t, \rho_{S}^{1,2}\right)=\frac{d}{d t} D\left(t, \rho_{S}^{1,2}\right)>0 .
$$

Consequently, the non-Markovianity of an open quantum system's dynamics $\{\Lambda(t)\}_{t \geqslant 0}$ is quantified by the measure

$$
\mathscr{N}=\max _{\rho_{S}^{1,2}} \int_{\Omega_{+}} \sigma\left(t, \rho_{S}^{1,2}\right) d t .
$$

The maximization involved in the definition of this measure can be greatly simplified since the optimal states must be orthogonal [22] and, even more, one can determine $\mathscr{N}$ by means of a local maximization over one state only [23]. This measure of non-Markovianity has been also investigated experimentally in all-optical settings [15,24,25].

\section{B. Divisibility measure}

The definition given by Rivas, Huelga, and Plenio (RHP) [6] identifies Markovian dynamics with those dynamics which are described by a CP-divisible family of quantum dynamical maps $\{\Lambda(t)\}_{t \geqslant 0}$ (CP standing for completely positive), i.e., such that

$$
\Lambda\left(t_{2}\right)=\Lambda\left(t_{2}, t_{1}\right) \Lambda\left(t_{1}\right) \quad \forall t_{2} \geqslant t_{1} \geqslant 0,
$$

$\Lambda\left(t_{2}, t_{1}\right)$ being itself a completely positive map. Indeed, if $\Lambda\left(t_{2}, t_{1}\right)=\Lambda\left(t_{2}-t_{1}\right)$ the composition law in Eq. (9) is equivalent to the semigroup composition law. An important property of this definition is that, provided that the evolution of the reduced state can be formulated by a time-local master equation

$$
\begin{aligned}
\frac{d}{d t} \rho_{S}(t)= & \mathcal{K}(t)\left[\rho_{S}(t)\right]=-i\left[H(t), \rho_{S}(t)\right] \\
& +\sum_{k} \gamma_{k}(t)\left(L_{k}(t) \rho_{S}(t) L_{k}^{\dagger}(t)\right. \\
& \left.-\frac{1}{2}\left\{L_{k}^{\dagger}(t) L_{k}(t), \rho_{S}(t)\right\}\right),
\end{aligned}
$$

the positivity of the coefficients, $\gamma_{k}(t) \geqslant 0$ for any $t \geqslant 0$, is equivalent to the $\mathrm{CP}$ divisibility of the corresponding dynamics. This can be shown by taking into account the family of propagators $\Lambda\left(t_{2}, t_{1}\right)$ associated with Eq. (10),

$$
\Lambda\left(t_{2}, t_{1}\right)=T_{\leftarrow} \exp \left(\int_{t_{1}}^{t_{2}} \mathcal{K}(s) d s\right),
$$

where $T_{\leftarrow}$ denotes the time ordering and $\Lambda(t, 0) \equiv \Lambda(t)$. By construction, the propagators $\Lambda\left(t_{2}, t_{1}\right)$ satisfy Eq. (9), but, in general, they are not CP maps. One can show [26,27] that the propagators are actually $\mathrm{CP}$ if and only if the coefficients $\gamma_{k}(t)$ are positive functions of time. 

by

The corresponding measure of non-Markovianity is given

$$
\mathcal{I}=\int_{\mathbb{R}^{+}} d t \mathfrak{g}(t)
$$

with

$$
\mathfrak{g}(t)=\lim _{\epsilon \rightarrow 0^{+}} \frac{\frac{1}{N}\left\|\Lambda_{\text {Choi }}(t, t+\epsilon)\right\|_{1}-1}{\epsilon},
$$

where $\Lambda_{\text {Choi }}$ is the Choi matrix associated with $\Lambda$. Given a maximally entangled state between the system and an ancilla, $|\psi\rangle=\frac{1}{\sqrt{N}} \sum_{k=1}^{N}\left|u_{k}\right\rangle \otimes\left|u_{k}\right\rangle$, one has [28]

$$
\Lambda_{\text {Choi }}=N\left(\Lambda \otimes \mathbb{1}_{N}\right)(|\psi\rangle\langle\psi|) .
$$

The positivity of the Choi matrix corresponds to the complete positivity of the map $\Lambda$ and it is equivalent to the condition $\left\|\Lambda_{\text {Choi }}\right\|_{1}=N$, so that the quantity $\mathfrak{g}(t)$ is different from zero if and only if the CP divisibility of the dynamics is broken.

Finally, since the trace distance is contractive under CPT maps, if a dynamics is Markovian according to the RHP definition, then it is so also according to the BLP definition, i.e.,

$$
\mathcal{I}=0 \Longrightarrow \mathscr{N}=0
$$

while the opposite implication does not hold [26,29,30].

\section{THE QUANTUM REGRESSION THEOREM}

As recalled in the Introduction, the quantum regression theorem provides a benchmark structure in order to study the multitime correlation functions of an open quantum system. For the sake of simplicity, we focus on the two-time correlation functions only. Given two open system operators, $A \otimes \mathbb{1}_{E}$ and $B \otimes \mathbb{1}_{E}$, where $\mathbb{1}_{E}$ denotes the identity on the Hilbert space associated with the environment, their two-time correlation function is defined as

$$
\begin{aligned}
\left\langle A\left(t_{2}\right) B\left(t_{1}\right)\right\rangle \equiv & \operatorname{Tr}\left[U^{\dagger}\left(t_{2}\right) A \otimes \mathbb{1}_{E} U\left(t_{2}\right)\right. \\
& \left.\times U^{\dagger}\left(t_{1}\right)\left(B \otimes \mathbb{1}_{E}\right) U\left(t_{1}\right) \rho_{S E}(0)\right],
\end{aligned}
$$

where $U(t)$ is the overall unitary evolution operator and we set $t_{2} \geqslant t_{1} \geqslant 0$. In the following, we assume an initial state as in Eq. (1), as well as a time-independent total Hamiltonian $H_{T}=H_{S} \otimes \mathbb{1}_{E}+\mathbb{1}_{S} \otimes H_{E}+H_{I}$, so that $U(t)=e^{-i H_{T} t}$.

The condition of an initial product state with a fixed environmental state guarantees the existence of a reduced dynamics; see Eqs. (1) and (2). This means that all the one-time probabilities associated with the observables of the open systems and, as a consequence, their mean values can be evaluated by means of the family of reduced dynamical maps only, without need for any further reference to the overall unitary dynamics. An analogous result holds for the two-time correlation functions, if one can apply the so-called quantum regression theorem. The latter essentially states that under proper conditions the dynamics of the two-time correlation functions can be reconstructed from the dynamics of the mean values, or, equivalently, of the statistical operator. Indeed, if the quantum regression theorem cannot be applied, one needs to come back to the full unitary dynamics in order to determine the evolution of the two-time correlation functions. We will not repeat here the detailed derivation of the quantum regression theorem, which can be found in [13,14,31]. Nevertheless, let us recall the basic ideas. First, by introducing the operator

$$
\chi\left(t_{2}, t_{1}\right)=e^{-i H_{T}\left(t_{2}-t_{1}\right)} B \otimes \mathbb{1}_{E} \rho_{S E}\left(t_{1}\right) e^{i H_{T}\left(t_{2}-t_{1}\right)},
$$

the two-time correlation function in Eq. (16) can be rewritten as

$$
\left\langle A\left(t_{2}\right) B\left(t_{1}\right)\right\rangle=\operatorname{Tr}_{S} A \operatorname{Tr}_{E} \chi\left(t_{2}, t_{1}\right) .
$$

Now, suppose that we can describe the evolution of $\chi\left(t_{2}, t_{1}\right)$ with respect to $t_{2}$ with the same dynamical maps which fix the evolution of the statistical operator, i.e.,

$$
\chi\left(t_{2}, t_{1}\right)=\Lambda\left(t_{2}, t_{1}\right)\left[\chi\left(t_{1}, t_{1}\right)\right],
$$

where $\Lambda\left(t_{2}, t_{1}\right)$ is the propagator introduced in Eq. (11). Then, Eq. (18) directly provides

$$
\left\langle A\left(t_{2}\right) B\left(t_{1}\right)\right\rangle_{q r t}=\operatorname{Tr}_{S} A \Lambda\left(t_{2}, t_{1}\right)\left[B \rho_{S}\left(t_{1}\right)\right] .
$$

The two-time correlation functions can be fully determined by the dynamical maps which fix the evolution of the statistical operator: the validity of Eq. (20) can be identified with the validity of the quantum regression theorem and we will use the subscript $q r t$ to denote the two-time correlation functions evaluated through Eq. (20). Indeed, all the procedure relies on Eq. (19), which requires that the same assumptions made in order to derive the dynamics of $\rho_{S}(t)$ can be made also to get the evolution of $\chi\left(t_{2}, t_{1}\right)$ with respect to $t_{2}$ [14]. Especially, the hypothesis of an initial total product state in Eq. (1) turns into the hypothesis of a product state at any intermediate time $t_{1}$,

$$
\rho_{S E}\left(t_{1}\right)=\rho_{S}\left(t_{1}\right) \otimes \rho_{E} .
$$

The physical idea is that the quantum regression theorem holds when the system-environment correlations due to the interaction can be neglected [32]. Note that this condition will never be strictly satisfied, as long as the system and the environment mutually interact, but it should be understood as a guideline to detect the regimes in which Eq. (20) provides a satisfying description of the evolution of the two-time correlation functions. More precisely, Dümcke [33] demonstrated that the exact expression of the two-time (multitime) correlation functions, see Eq. (16), converges to the expression in Eq. (20) in the weak coupling limit and in the singular coupling limit. As is well known, in these limits the reduced dynamics converges to a semigroup dynamics [34,35]. Nevertheless, the correctness of a semigroup description of the reduced dynamics is not always enough to guarantee the accuracy of the quantum regression theorem [36,37]. More in general, the precise link between a sharply defined notion of Markovianity of quantum dynamics and the quantum regression theorem has still to be investigated.

The quantum regression theorem provided by Eq. (20) can be equivalently formulated in terms of the differential equations satisfied by mean values and two-time correlation functions, as was originally done in [38]. For the sake of simplicity, let us restrict ourselves to the finite-dimensional case, i.e., the Hilbert space associated with the open system is $\mathbb{C}^{N}$. Consider a reduced dynamics fixed by the family of maps $\{\Lambda(t)\}_{t \geqslant 0}$ and a basis $\left\{E_{i}\right\}_{1, \ldots, N^{2}}$ of linear operators on $\mathbb{C}^{N}$, such that the corresponding mean values fulfill the coupled 
linear equations of motion [31]

$$
\frac{d}{d t}\left\langle E_{i}(t)\right\rangle=\sum_{j} G_{i j}(t)\left\langle E_{j}(t)\right\rangle
$$

with the initial condition $\left.\left\langle E_{i}(t)\right\rangle\right|_{t=0}=\left\langle E_{i}(0)\right\rangle$. In this case, the quantum regression theorem is said to hold if the two-time correlation functions satisfy $[13,14]$

$$
\frac{d}{d t_{2}}\left\langle E_{i}\left(t_{2}\right) E_{k}\left(t_{1}\right)\right\rangle_{q r t}=\sum_{j} G_{i j}\left(t_{2}\right)\left\langle E_{j}\left(t_{2}\right) E_{k}\left(t_{1}\right)\right\rangle_{q r t},
$$

with the initial condition

$$
\left.\left\langle E_{i}\left(t_{2}\right) E_{k}\left(t_{1}\right)\right\rangle\right|_{t_{2}=t_{1}}=\left\langle E_{i}\left(t_{1}\right) E_{k}\left(t_{1}\right)\right\rangle .
$$

In the following, we will compare the evolution of the exact two-time correlation functions obtained from the full unitary evolution $\left\langle E_{i}\left(t_{2}\right) E_{k}\left(t_{1}\right)\right\rangle$, see Eq. (16), with those predicted by the quantum regression theorem $\left\langle E_{i}\left(t_{2}\right) E_{k}\left(t_{1}\right)\right\rangle_{q r t}$. To quantify the error made by using the latter, we exploit the relative error; i.e., we use the following figure of merit:

$$
Z \equiv\left|1-\frac{\left\langle A\left(t_{2}\right) B\left(t_{1}\right)\right\rangle_{q r t}}{\left\langle A\left(t_{2}\right) B\left(t_{1}\right)\right\rangle}\right|
$$

which depends on the chosen couple of open system operators. Hence, in general, one should consider different estimators, one for each couple of operators in the basis $\left\{E_{i}\right\}_{1, \ldots, N^{2}}$, and a maximization over them could be taken. Nevertheless, in the following analysis it will be enough to deal with a single couple of system operators, which fully encloses the violations of the quantum regression theorem for the models at hand.

\section{PURE-DEPHASING SPIN BOSON MODEL}

In this section, we take into account a model whose full unitary evolution can be exactly evaluated [13,39], so as to obtain the exact expression of the two-time correlation functions, to be compared with the expression provided by the quantum regression theorem. This model is a pure-decoherence model, in which the decay of the coherences occurs without a decay of the corresponding populations. Indeed, this is due to the fact that the free Hamiltonian of the open system $H_{S} \otimes \mathbb{1}_{E}$ commutes with the total Hamiltonian $H_{T}$ [13].

\section{A. The model}

Let us consider a two-level system linearly interacting with a bath of harmonic oscillators, so that the total Hamiltonian is

$$
\begin{aligned}
H_{T}= & \frac{\omega_{s}}{2} \sigma_{z} \otimes \mathbb{1}_{E}+\mathbb{1}_{S} \otimes \sum_{k} \omega_{k} b_{k}^{\dagger} b_{k} \\
& +\sum_{k} \sigma_{z} \otimes\left(g_{k} b_{k}^{\dagger}+g_{k}^{*} b_{k}\right) .
\end{aligned}
$$

The unitary evolution operator of the overall system in the interaction picture is given by [13]

$$
U(t)=e^{i \Psi(t)} V(t)
$$

where the first factor is an irrelevant global phase and the second factor is the unitary operator

$$
V(t)=\exp \left[\frac{1}{2} \sigma_{z} \otimes \sum_{k}\left(\alpha_{k}(t) b_{k}^{\dagger}-\alpha_{k}^{*}(t) b_{k}\right)\right],
$$

with

$$
\alpha_{k}(t)=2 g_{k} \frac{1-e^{i \omega_{k} t}}{\omega_{k}} .
$$

The reduced dynamics is readily calculated to give

$$
\rho_{S}(t)=\left(\begin{array}{cc}
\rho_{00} & \rho_{01} \gamma(t) e^{-i \omega_{s} t} \\
\rho_{10} \gamma^{*}(t) e^{i \omega_{s} t} & \rho_{11}
\end{array}\right),
$$

where the function $\gamma(t)$ is given by

$$
\begin{aligned}
\gamma(t) & =\operatorname{Tr}_{E} \rho_{E} \prod_{k} \exp \left[\alpha_{k}(t) b_{k}^{\dagger}-\alpha_{k}^{*}(t) b_{k}\right] \\
& =\operatorname{Tr}_{E} \rho_{E} \prod_{k} \Delta\left(\alpha_{k}(t)\right),
\end{aligned}
$$

$\Delta(\alpha)$ being the displacement operator of argument $\alpha$ [40]. The associated master equation reads

$$
\frac{d}{d t} \rho_{S}(t)=-i \frac{\epsilon(t)}{2}\left[\sigma_{z}, \rho_{S}(t)\right]+\frac{\mathcal{D}(t)}{2}\left[\sigma_{z} \rho_{S}(t) \sigma_{z}-\rho_{S}(t)\right],
$$

where

$$
\epsilon(t)=\omega_{s}-\operatorname{Im}\left[\frac{d \gamma(t) / d t}{\gamma(t)}\right]
$$

and the so-called dephasing function $\mathcal{D}(t)$ is

$$
\mathcal{D}(t)=-\operatorname{Re}\left[\frac{d \gamma(t) / d t}{\gamma(t)}\right]=-\frac{d}{d t} \ln |\gamma(t)| .
$$

In the following, we will focus on the case of an initial thermal state of the bath, $\rho_{E}=\exp \left(-\beta H_{E}\right) / Z$ with $Z=$ $\operatorname{Tr}_{E} \exp \left(-\beta H_{E}\right)$ and $\beta=\left(k_{B} T\right)^{-1}$ the inverse temperature. We also consider the continuum limit: given a frequency distribution $f(\omega)$ of the bath modes, we introduce the spectral density $J(\omega)=4 f(\omega)|g(\omega)|^{2}$, so that one has [13]

$$
\gamma(t)=\exp \left[-\int_{0}^{\infty} d \omega J(\omega) \operatorname{coth}\left(\frac{\beta \omega}{2}\right) \frac{1-\cos (\omega t)}{\omega^{2}}\right],
$$

and hence $\epsilon(t)=\omega_{s}$ and

$$
\mathcal{D}(t)=\int_{0}^{\infty} d \omega J(\omega) \operatorname{coth}\left(\frac{\beta \omega}{2}\right) \frac{\sin (\omega t)}{\omega} .
$$

\section{B. Measures of non-Markovianity}

\section{General expressions}

For this specific model, the two definitions of Markovianity are actually equivalent [41]; i.e., not only Eq. (15) holds, but also the opposite does so. This is due to the fact that there is only one operator contribution in the time-local master equation (31), corresponding to the dephasing interaction. Nevertheless, the numerical values of the two measures of nonMarkovianity are in general different and, more importantly, they depend in a different way on the parameters of the model. 
Let us start by evaluating the BLP measure; see Sec. II A. The trace distance between two reduced states evolved through Eq. (29) is given by

$$
D\left(t, \rho_{S}^{1,2}\right)=\sqrt{\delta_{p}^{2}+\left|\delta_{c}\right|^{2}|\gamma(t)|^{2}},
$$

where $\delta_{p}=\rho_{00}^{1}-\rho_{00}^{2}$ and $\delta_{c}=\rho_{01}^{1}-\rho_{01}^{2}$ are the differences between, respectively, the populations and the coherences of the two initial conditions $\rho_{S}^{1}$ and $\rho_{S}^{2}$. The couple of initial states that maximizes the growth of the trace distance is given by the pure orthogonal states $\rho_{S}^{1,2}=\left|\psi_{ \pm}\right\rangle\left\langle\psi_{ \pm}\right|$, where $\left|\psi_{ \pm}\right\rangle=$ $\frac{1}{\sqrt{2}}(|0\rangle \pm|1\rangle)$, and the corresponding trace distance at time $t$ is simply $|\gamma(t)|$. The BLP measure therefore reads

$$
\mathscr{N}=\sum_{m}\left[\left|\gamma\left(b_{m}\right)\right|-\left|\gamma\left(a_{m}\right)\right|\right],
$$

where $\Omega_{+}=\bigcup_{m}\left(a_{m}, b_{m}\right)$ is the union of the time intervals in which $|\gamma(t)|$ increases. The BLP measure is different from zero if and only if $d|\gamma(t)| / d t>0$ for some interval of time, which is equivalent to the requirement that the dephasing function $\mathcal{D}(t)$ in Eq. (31) is not a positive function of time, i.e., that the CP divisibility of the dynamics is broken; Sec. II B. As anticipated, for this model $\mathscr{N}>0 \Longleftrightarrow \mathscr{I}>0$. Furthermore, given a pure dephasing master equation as in Eq. (31), one has [6] $\mathfrak{g}(t)=0$ if $\mathcal{D}(t) \geqslant 0$ and $\mathfrak{g}(t)=-\mathcal{D}(t)$ if $\mathcal{D}(t)<0$, so that, see Eq. (33),

$$
\mathscr{I}=\sum_{m}\left[\ln \left|\gamma\left(b_{m}\right)\right|-\ln \left|\gamma\left(a_{m}\right)\right|\right],
$$

where the $a_{m}$ and $b_{m}$ are defined as for the BLP measure.

\section{Zero-temperature environment}

In order to evaluate explicitly the non-Markovianity measures, we need to specify the spectral density $J(\omega)$. In the following, we assume a spectral density of the form

$$
J(\omega)=\lambda \frac{\omega^{s}}{\Omega^{s-1}} e^{-\frac{\omega}{\Omega}},
$$

where $\lambda$ is the coupling strength, the parameter $s$ fixes the low-frequency behavior, and $\Omega$ is a cutoff frequency. The nonMarkovianity for the pure dephasing spin model with a spectral density as in Eq. (39) has been considered in $[18,42]$ for the case $\lambda=1$. We are now interested in the comparison between non-Markovianity and violations of the quantum regression theorem, so that, as will become clear in the next section, the dependence on $\lambda$ plays a crucial role. In particular, we consider the case of low temperature, i.e., $\beta \gg 1$, so that $\operatorname{coth}\left(\frac{\beta \omega}{2}\right) \approx 1$. The dephasing function in this case reads, see Eq. (35),

$$
\mathcal{D}_{s}(t)=\frac{\lambda \Omega \Gamma(s)}{\left[1+(\Omega t)^{2}\right]^{\frac{s}{2}}} \sin [s \arctan (\Omega t)],
$$

with $\Gamma(s)$ the Euler gamma function, which can be expressed in the equivalent but more compact form, see the Appendix,

$$
\mathcal{D}_{s}(t)=\lambda \Omega \Gamma(s) \frac{\operatorname{Im}\left[(1+i \Omega t)^{s}\right]}{\left[1+(\Omega t)^{2}\right]^{s}} .
$$

Correspondingly, the decoherence function can be written as

$$
\gamma_{s}(t)=\exp \left[-\lambda \Gamma(s-1)\left(1-\frac{\operatorname{Re}\left[(1+i \Omega t)^{s-1}\right]}{\left[1+(\Omega t)^{2}\right]^{s-1}}\right)\right] .
$$

As before, let $\Omega_{+}$be the union of the time intervals for which $\mathcal{D}(t)<0$, i.e., equivalently, $|\gamma(t)|$ increases. The number of solutions of the equation $\mathcal{D}(t)=0$ grows with the parameter $s$ : for $s=1,2$ the dephasing function is always strictly positive, while for $s=3$ and $s=4$ there is one zero at $t_{3}^{*}=\frac{\sqrt{3}}{\Omega}$ and $t_{4}^{*}=\frac{1}{\Omega}$, respectively. Indeed, if the number of zeros is odd, $\mathcal{D}(t)$ is negative from its last zero to infinity, while if the number of zeros is even, it approaches zero asymptotically from above. As a consequence, the two measures of non-Markovianity are equal to zero for $s=1,2$ and, to give an example, one has for $s=3$

$$
\begin{aligned}
& \mathscr{N}_{3}(\lambda)=\lim _{t \rightarrow \infty}|\gamma(t)|-\left|\gamma\left(t_{3}^{*}\right)\right|=e^{-\lambda}-e^{-\frac{9}{8} \lambda}, \\
& \mathscr{I}_{3}(\lambda)=\lim _{t \rightarrow \infty} \ln |\gamma(t)|-\ln \left|\gamma\left(t_{3}^{*}\right)\right|=\frac{\lambda}{8},
\end{aligned}
$$

and, analogously, for $s=4$

$$
\mathscr{N}_{4}(\lambda)=e^{-2 \lambda}-e^{-\frac{5}{2} \lambda}, \quad \mathscr{I}_{4}(\lambda)=\frac{\lambda}{2} .
$$

In Figs. 1(a) and 1(b), we report, respectively, the BLP and the RHP measures of non-Markovianity as a function of $\lambda$, for different values of $s$.

The behavior of the two measures is clearly different. The RHP measure is a monotonically increasing function of both

(a)

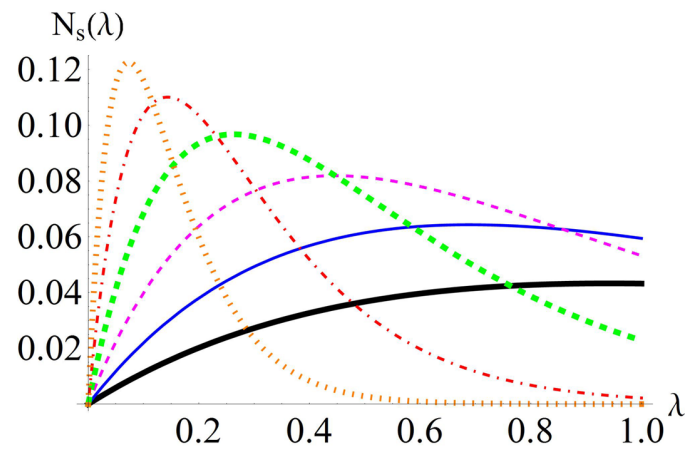

(b)

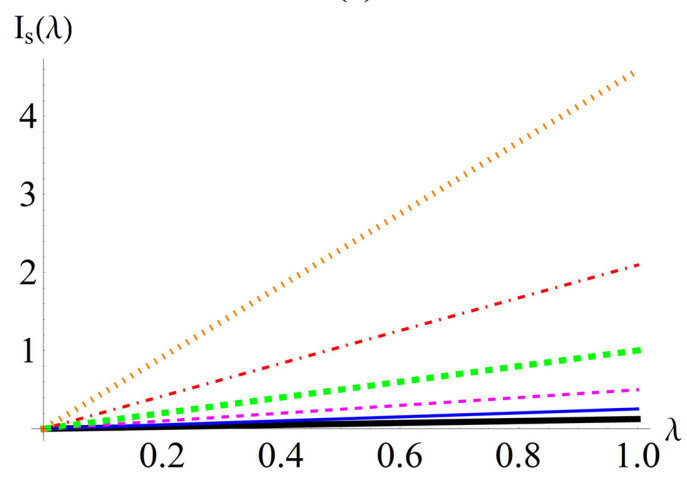

FIG. 1. (Color online) (a) BLP measure of non-Markovianity $\mathscr{N}_{s}(\lambda)$, see Eq. (37), and (b) RHP measure of non-Markovianity $\mathscr{I}_{s}(\lambda)$, see Eq. (38), as a function of the coupling strength $\lambda$ for increasing values of the parameter $s$. In both panels the curves are evaluated for $s=3$ (black thick solid line), $s=3.5$ (blue solid line), $s=4$ (magenta dashed line), $s=4.5$ (green dashed thick line), $s=5$ (red dot-dashed line), and $s=5.5$ (orange dotted line). 
(a)

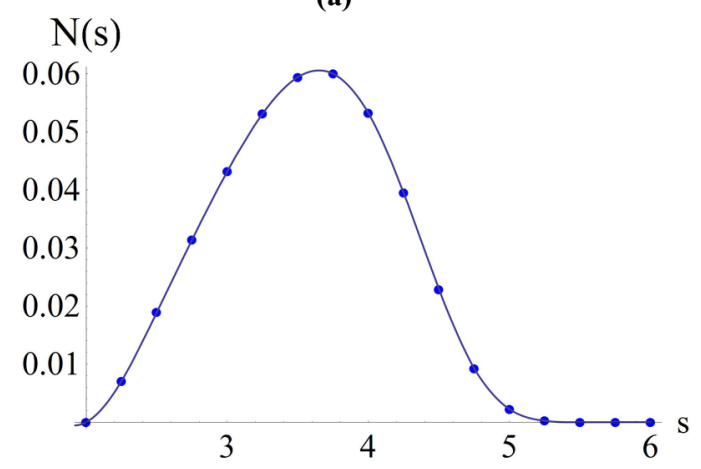

(b)

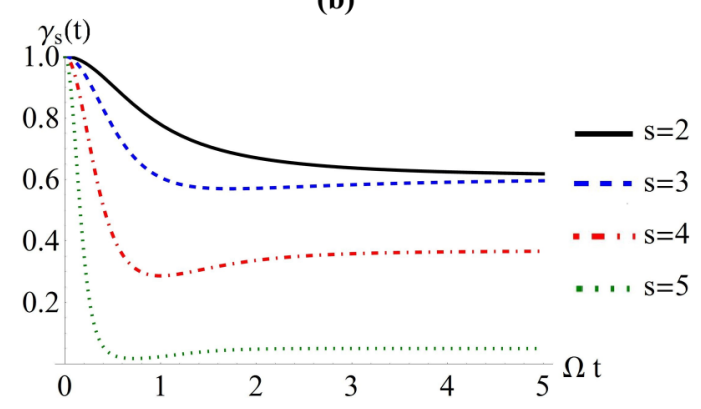

(c)

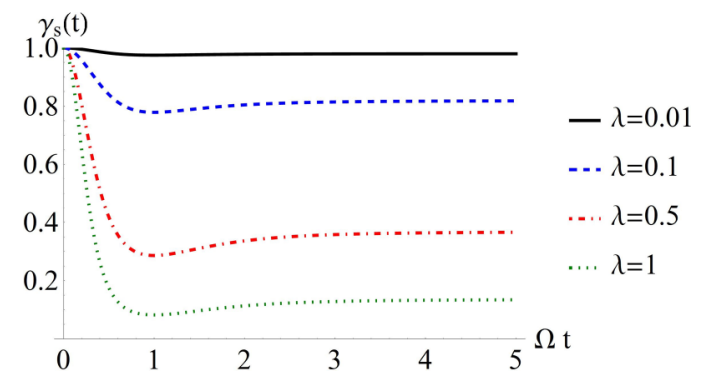

FIG. 2. (Color online) (a) BLP measure of non-Markovianity $\mathscr{N}_{s}(\lambda)$, see Eq. (37), as a function of the parameter $s$, for $\lambda=1$. (b) and (c) Decoherence function $\gamma_{s}(t)$ as a function of rescaled for $\lambda=0.5$ and different values of $s$ (b), and for $s=4$ and different values of $\lambda$ (c).

$\lambda$ and $s$ : the increase is linear with respect to the former parameter and exponential with respect to the latter. On the other hand, for every fixed $s$, there is a critical value of the coupling strength $\lambda^{*}(s)$, which is smaller for increasing $s$, that separates two different regimes of the BLP measure: for $\lambda<\lambda^{*}(s)$, the non-Markovianity measure increases with the increase of the system-environment coupling, while for $\lambda>\lambda^{*}(s)$ it decreases with the increase of the coupling. Analogously, there is a threshold value $s^{*}(\lambda)$ of the parameter $s$, which is higher for smaller values of $\lambda$, such that the BLP measure increases for $s<s^{*}(\lambda)$ and decreases for $s>s^{*}(\lambda)$; see also Fig. 2(a). Incidentally, the maximum value as a function of $\lambda, \max _{\lambda} \mathscr{N}_{s}(\lambda)$, is a monotonically increasing function of the parameter $s$. Indeed, the different behavior of the non-Markovianity measures traces back to their different functional dependence of the decoherence function $\gamma_{s}(t)$, which is plotted in Figs. 2(b) and 2(c) for different values of $s$ and $\lambda$. One can see how $\gamma_{s}(t)$ takes on smaller values within $[0,1]$ for growing values of $\lambda$, while its global minimum decreases with increasing $s$. Now, while the BLP measure is fixed by the difference between the values of $\gamma_{s}(t)$ at the edges of the time intervals $\left[a_{m}, b_{m}\right]$ in which $\gamma_{s}(t)$ increases, see Eq. (37), the RHP measure is fixed by the ratio between the same values, see Eq. (38). Hence, as the coupling strength grows over the threshold $\lambda^{*}(s)$ or the parameter $s$ overcomes the threshold $s^{*}(\lambda)$, the difference between $b_{m}$ and $a_{m}$ is increasingly smaller, and therefore $\mathscr{N}_{s}(\lambda)$ is so. However, the ratio between $b_{m}$ and $a_{m}$ always increases with $\lambda$ and $s$, as witnessed by the corresponding monotonic increase of $\mathscr{I}_{s}(\lambda)$.

\section{Validity of regression hypothesis}

\section{Exact expression versus quantum regression theorem}

The exact unitary evolution, Eq. (26), directly provides us with the average values, as well as the two-time correlation functions of the observables of the system. In view of the comparison with the description given by the quantum regression theorem, see Sec. III, let us focus on the basis of linear operators on $\mathbb{C}^{2}$, orthonormal with respect to the HilbertSchmidt scalar product, given by $\left\{\mathbb{1} / \sqrt{2}, \sigma_{-}, \sigma_{+}, \sigma_{z} / \sqrt{2}\right\}$. Indeed, the first and the last elements of the basis are constant of motion, see Eq. (29), while the mean values of $\sigma_{-}$and $\sigma_{+}$ evolve according to, respectively,

$$
\left\langle\sigma_{-}(t)\right\rangle=\gamma(t) e^{-i \omega_{s} t}\left\langle\sigma_{-}(0)\right\rangle
$$

and the complex conjugate relation. In a similar way, all the two-time correlation functions involving $\mathbb{1} / \sqrt{2}$ or $\sigma_{z} / \sqrt{2}$ satisfy the condition of the quantum regression theorem in a trivial way, as at most one operator within the two-time correlation function actually evolves. The only nontrivial expressions are thus the following:

$$
\begin{aligned}
& \left\langle\sigma_{-}\left(t_{2}\right) \sigma_{+}\left(t_{1}\right)\right\rangle=e^{-i \omega_{s}\left(t_{2}-t_{1}\right)} \gamma\left(t_{2}, t_{1}\right) e^{i \phi\left(t_{2}, t_{1}\right)}\left\langle\left(\sigma_{-} \sigma_{+}\right)\left(t_{1}\right)\right\rangle, \\
& \left\langle\sigma_{+}\left(t_{2}\right) \sigma_{-}\left(t_{1}\right)\right\rangle=e^{i \omega_{s}\left(t_{2}-t_{1}\right)} \gamma^{*}\left(t_{2}, t_{1}\right) e^{i \phi\left(t_{2}, t_{1}\right)}\left\langle\left(\sigma_{+} \sigma_{-}\right)\left(t_{1}\right)\right\rangle,
\end{aligned}
$$

where

$$
\gamma\left(t_{2}, t_{1}\right)=\operatorname{Tr}_{E} \rho_{E} \prod_{k} \Delta\left(\alpha_{k}\left(t_{2}\right)-\alpha_{k}\left(t_{1}\right)\right)
$$

and

$$
\phi\left(t_{2}, t_{1}\right)=\sum_{k} \operatorname{Im}\left[\alpha_{k}^{*}\left(t_{2}\right) \alpha_{k}\left(t_{1}\right)\right] .
$$

Here, to derive (46) we used the properties of the displacement operator [40]

$$
\Delta(\alpha) \Delta(\beta)=\Delta(\alpha+\beta) e^{i \operatorname{Im}\left(\alpha \beta^{*}\right)}, \quad \Delta^{\dagger}(\alpha)=\Delta(-\alpha),
$$

and the equality $\left\langle\left(\sigma_{+} \sigma_{-}\right)(t)\right\rangle=\left\langle\sigma_{+} \sigma_{-}\right\rangle$.

We can now obtain the corresponding two-time correlation functions as predicted by the quantum regression theorem. By Eq. (45), one has

$$
\frac{d}{d t}\left\langle\sigma_{-}(t)\right\rangle=\left(\frac{d \gamma(t) / d t}{\gamma(t)}-i \omega_{s}\right)\left\langle\sigma_{-}(t)\right\rangle
$$

and the complex conjugate relation for $\left\langle\sigma_{+}(t)\right\rangle$. The specific choice of the operator basis has lead us to a diagonal matrix $G$ 
in Eq. (22). Hence, one has immediately

$$
\begin{aligned}
\left\langle\sigma_{-}\left(t_{2}\right) \sigma_{+}\left(t_{1}\right)\right\rangle_{q r t} & =e^{-i \omega_{s}\left(t_{2}-t_{1}\right)} \frac{\gamma\left(t_{2}\right)}{\gamma\left(t_{1}\right)}\left\langle\sigma_{-}\left(t_{1}\right) \sigma_{+}\left(t_{1}\right)\right\rangle, \\
\left\langle\sigma_{+}\left(t_{2}\right) \sigma_{-}\left(t_{1}\right)\right\rangle_{q r t} & =e^{i \omega_{s}\left(t_{2}-t_{1}\right)} \frac{\gamma^{*}\left(t_{2}\right)}{\gamma^{*}\left(t_{1}\right)}\left\langle\sigma_{+}\left(t_{1}\right) \sigma_{-}\left(t_{1}\right)\right\rangle .
\end{aligned}
$$

The quantum regression theorem will be generally violated within this model; compare Eq. (46) and (50). We quantify such a violation by means of the figure of merit introduced in Eq. (24), which for the couple of operators $\sigma_{-}$and $\sigma_{+}$reads

$$
\begin{aligned}
Z & =\left|1-\frac{\left\langle\sigma_{-}\left(t_{2}\right) \sigma_{+}\left(t_{1}\right)\right\rangle_{q r t}}{\left\langle\sigma_{-}\left(t_{2}\right) \sigma_{+}\left(t_{1}\right)\right\rangle}\right| \\
& =\left|1-\frac{\gamma\left(t_{2}\right)}{\gamma\left(t_{1}\right) \gamma\left(t_{2}, t_{1}\right) e^{i \phi\left(t_{2}, t_{1}\right)}}\right| .
\end{aligned}
$$

\section{Quantitative analysis of the violations of the quantum regression theorem}

The expressions of the previous paragraph hold for generic initial state of the bath and spectral density. Now, we come back to the specific choice of an initial thermal bath. The results in Eq. (50) are in this case in agreement with those found in [43], where the two-time correlation functions have been evaluated focusing on a spectral density as in Eq. (39) with $s=1$, while keeping a generic temperature of the bath. Instead, we will focus on the case $T=0$ and maintain a generic value of $s$ in order to compare the behavior of the two-time correlation functions with the measures of non-Markovianity.

First, note that by using the definition of the displacement operator as well as Eq. (28), one can show the general identity

$$
\Delta\left(\alpha_{k}\left(t_{2}\right)-\alpha_{k}\left(t_{1}\right)\right)=\Delta\left(\alpha_{k}\left(t_{2}-t_{1}\right) e^{i \omega_{k} t_{1}}\right) .
$$

But then, since for a thermal state $\operatorname{Tr}_{E} \Delta(\alpha) \rho_{E}$ is a function of $|\alpha|$ only [13], Eq. (52) implies

$$
\gamma\left(t_{2}, t_{1}\right)=\gamma\left(t_{2}-t_{1}\right)
$$

see Eqs. (47) and (30). In addition we have in the continuum limit, see Eq. (48),

$$
\phi\left(t_{2}, t_{1}\right)=\int d \omega \frac{J(\omega)}{\omega^{2}}\left\{\sin \left(\omega t_{2}\right)-\sin \left(\omega t_{1}\right)-\sin \left[\omega\left(t_{2}-t_{1}\right)\right]\right\},
$$

so that, for $J(\omega)$ as in Eq. (39) and using Eq. (35) in the zero-temperature limit, we get

$$
\phi_{s}\left(t_{2}, t_{1}\right)=\left[\mathcal{D}_{s-1}\left(t_{2}\right)-\mathcal{D}_{s-1}\left(t_{1}\right)-\mathcal{D}_{s-1}\left(t_{2}-t_{1}\right)\right] / \Omega .
$$

The identities in Eqs. (41) and (42), along with Eqs. (53) and (54), finally provide us with the explicit expression of the estimator for the violations of the quantum regression theorem, see Eq. (51),

$$
\begin{aligned}
Z_{s}(\lambda)= & \mid 1-\exp \left(\lambda \Gamma ( s - 1 ) \left\{1-\left[1+i \Omega\left(t_{2}-t_{1}\right)\right]^{1-s}\right.\right. \\
& \left.\left.-\left(1+i \Omega t_{1}\right)^{1-s}+\left(1+i \Omega t_{2}\right)^{1-s}\right\}\right) \mid,
\end{aligned}
$$

whose behavior as a function of $\lambda$ and $s$ is shown in Figs. 3(a) and $3(\mathrm{~b})$. The violation of the quantum regression theorem monotonically increases with increasing values of both the coupling strength $\lambda$ and the parameter $s$. This behavior is (a)

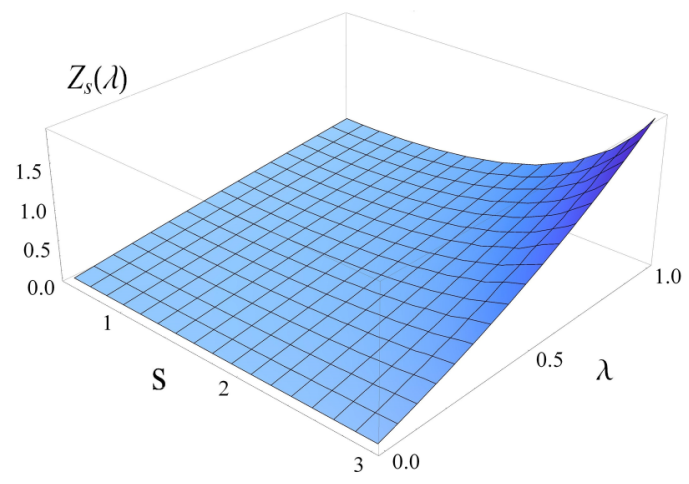

(b)

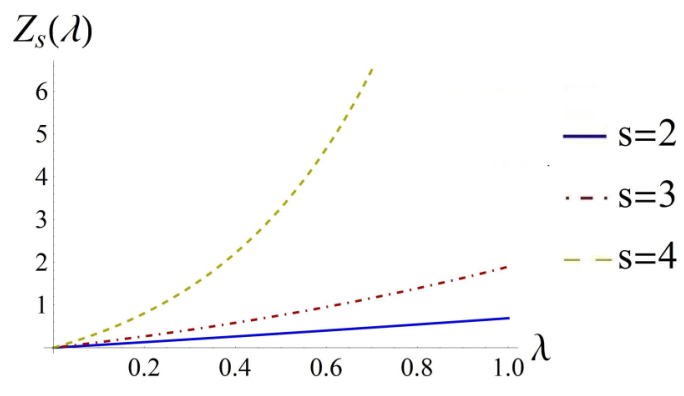

FIG. 3. (Color online) (a) $Z_{s}(\lambda)$ as a function of the parameter $s$ and of the coupling strength $\lambda$, see Eq. (55), for $\Omega t_{1}=1$ and $\Omega t_{2}=2$. (b) Section of (a) for $s=2,3,4$.

clearly in agreement with that of the RHP measure of nonMarkovianity; see Sec. IV B 2 and in particular Fig. 1. From a quantitative point of view there is, however, some difference as the estimator $Z_{s}(\lambda)$, at variance with the RHP measure, grows linearly with $\lambda$ only for small values of $s$, while it growths faster for $s>3$; compare with Fig. 1(b). In any case, the RHP measure appears to be more directly related with the strength of the violation to the quantum regression theorem, as compared with the BLP measure. This can be traced back to the different influence of the system-environment correlations on the two measures. As we recalled in Sec. III, the hypothesis that the state of the total system at any time $t$ is well approximated by the product state between the state of the open system and the initial state of the environment, see Eq. (21), lies at the basis of the quantum regression theorem. This hypothesis is expected to hold in the weak coupling regime, while for an increasing value of $\lambda$, the interaction will build stronger system-environment correlations, leading to a strong violation of the quantum regression theorem. The establishment of correlations between the system and the environment due to the interaction plays a significant role also in the subsequent presence of memory effects in the dynamics of the open system [44-46]. Indeed, different signatures of the memory effects can be affected by system-environment correlations in different ways. In particular, the CP divisibility of the dynamical maps appears to be a more fragile property than the contractivity of the trace distance and therefore it is more sensitive to the violations of the quantum regression theorem. Furthermore, it is worth noting that the estimator $Z_{s}(\lambda)$ steadily increases with the coupling strength $\lambda$ even for values of $s$ 
such that the corresponding reduced dynamics is Markovian according to either definitions. The validity of the quantum regression theorem calls therefore for stricter conditions than the Markovianity of quantum dynamics. In the case of the trace distance criterion for non-Markovianity, which is directly based on an observable quantity such as the distance in time between different system states, it is also important to stress the different dependence on $\lambda$ in quantifying non-Markovianity or violation of the quantum regression theorem, respectively. The monotonic dependence on the coupling strength in $Z_{s}(\lambda)$ is indeed not reflected in $\mathscr{N}(\lambda)$.

\section{PHOTONIC REALIZATION OF DEPHASING INTERACTION}

In the pure dephasing spin-boson model, there is no regime in which the quantum regression theorem is strictly satisfied, apart from the trivial case $\lambda=0$. In addition, we have shown that the strength of the violations of this theorem has the same qualitative behavior of the RHP non-Markovianity measure, as they increase with both $\lambda$ and the parameter $s$. In this section, we take into account a different pure dephasing model, which allows us to deepen our analysis on the relationship between the quantum regression theorem and the Markovianity of the reduced-system dynamics. In particular, we show that in general these two notions should be considered as different since the quantum regression theorem may be strongly violated, even if the open system's dynamics is Markovian, irrespective of the exploited definition.

\section{A. The model}

Let us deal with the pure-dephasing interaction considered in Ref. [15]. The open system here is represented by the polarization degrees of freedom of a photon generated by spontaneous parametric down conversion, while the environment consists in the corresponding frequency degrees of freedom. The overall unitary evolution, which is realized via a quartz plate that couples the polarization and frequency degrees of freedom, can be described as

$$
U(t)|j, \omega\rangle=e^{i n_{j} \omega t}|j, \omega\rangle \quad j=0,1,
$$

where $|0\rangle \equiv|H\rangle$ and $|1\rangle \equiv|V\rangle$ are the two polarization states (horizontal and vertical), with refractive indexes, respectively, $n_{0} \equiv n_{H}$ and $n_{1} \equiv n_{V}$, while $|\omega\rangle$ is the environmental state with frequency $\omega$. If we consider an initial product state, see Eq. (1), with a pure environmental state $\rho_{E}=\left|\Psi_{E}\right\rangle\left\langle\Psi_{E}\right|$, where

$$
\left|\Psi_{E}\right\rangle=\int d \omega f(\omega)|\omega\rangle
$$

we readily obtain that the reduced dynamics is given by Eq. (29). Again, we are in the presence of a pure dephasing dynamics, the only difference being the decoherence function, which now reads

$$
\gamma(t)=\int d \omega|f(\omega)|^{2} e^{i \Delta n \omega t},
$$

with $\Delta n \equiv n_{1}-n_{0}$. For the rest, the results of Secs. IV A and IV B directly apply also to this model: the master equation is given by Eq. (31), with $\epsilon(t)$ and $\mathcal{D}(t)$ as in, respectively,
Eq. (32) (for $\omega_{s}=0$ ) and Eq. (33), while the non-Markovianity measures are as in Eq. (37) and Eq. (38). Analogously, the two-time correlation functions are given by Eq. (46) with

$$
\gamma\left(t_{2}, t_{1}\right)=\gamma\left(t_{2}-t_{1}\right) \quad \phi\left(t_{2}, t_{1}\right)=0,
$$

while the application of the quantum regression theorem leads to the expressions in Eq. (50) (with $\omega_{s}=0$ ). Hence, the violations of the quantum regression theorem can be quantified by

$$
Z=\left|1-\frac{\left\langle\sigma_{-}\left(t_{2}\right) \sigma_{+}\left(t_{1}\right)\right\rangle_{q r t}}{\left\langle\sigma_{-}\left(t_{2}\right) \sigma_{+}\left(t_{1}\right)\right\rangle}\right|=\left|1-\frac{\gamma\left(t_{2}\right)}{\gamma\left(t_{1}\right) \gamma\left(t_{2}-t_{1}\right)}\right| .
$$

\section{B. Lorentzian frequency distributions}

\section{Semigroup dynamics}

Despite its great simplicity, this model allows us to describe the transition between Markovian and non-Markovian dynamics in concrete experimental settings [15,24]. Different dynamics are obtained for different choices of the initial environmental state, see Eq. (1) and the related discussion, i.e., for different initial frequency distributions, see Eq. (57). The latter can be experimentally set, e.g., by properly rotating a Fabry-Pérot cavity, through which a beam of photons generated by spontaneous parametric down conversion passes [15]. A natural benchmark is represented by the Lorentzian distribution

$$
|f(\omega)|^{2}=\frac{\delta \omega}{\pi\left[\left(\omega-\omega_{0}\right)^{2}+(\delta \omega)^{2}\right]},
$$

where $\delta \omega$ is the width of the distribution and $\omega_{0}$ its central frequency, as this provides a reduced semigroup dynamics [46]. The decoherence function, which is given by the Fourier transform of the frequency distribution, see Eq. (58), is in fact

$$
\gamma(t)=e^{-\Delta n\left(\delta \omega-i \omega_{0}\right) t} .
$$

Thus, replacing this expression in Eqs. (32) and (33), one obtains a Lindblad equation, given by Eq. (31) with $\epsilon(t)=-\Delta n \omega_{0}$ and $\mathcal{D}(t)=\Delta n \delta \omega$. In addition, $\gamma\left(t_{2}-t_{1}\right)=$ $\gamma\left(t_{2}\right) / \gamma\left(t_{1}\right)$ and hence, as one can immediately see by Eq. (60), $Z=0$. For this model, as long as the reduced dynamics is determined by a completely positive semigroup, the quantum regression theorem is strictly valid. Let us emphasize that this is the case even if the total state is not a product state at any time $t$. For example if the initial state of the open system is the pure state $\left|\psi_{S}\right\rangle=\alpha|H\rangle+\beta|V\rangle$, with $|\alpha|^{2}+|\beta|^{2}=1$, the total state at time $t$ is

$\left|\psi_{S E}(t)\right\rangle=\int d \omega f(\omega)\left(\alpha e^{i n_{H} \omega t}|H, \omega\rangle+\beta e^{i n_{V} \omega t}|V, \omega\rangle\right)$.

This is an entangled state, of course unless $\alpha=0$ or $\beta=0$; nevertheless, the quantum regression theorem does hold. This clearly shows that for the quantum regression theorem, as for the semigroup description of the dynamics [45-47], the approximation encoded in Eq. (21) should be considered as an effective description of the total state, which can be very different from its actual form, even when the theorem is valid. 


\section{Time-inhomogeneous Markovian and non-Markovian dynamics}

Now, we consider a more general class of frequency distributions; namely, the linear combination of two Lorentzian distributions,

$$
|f(\omega)|^{2}=\sum_{j=1,2} \frac{A_{j} \delta \omega_{j}}{\pi\left[\left(\omega-\omega_{0, j}\right)^{2}+\left(\delta \omega_{j}\right)^{2}\right]},
$$

with $A_{1}+A_{2}=1$. The decoherence function (58) is in this case

$$
\gamma(t)=\frac{e^{-\Delta n\left(\delta \omega_{1}-i \omega_{0,1}\right) t}+r e^{-\Delta n\left(\delta \omega_{2}-i \omega_{0,2}\right) t}}{1+r},
$$

with $r \equiv \frac{A_{2}}{A_{1}}$, while the estimator of the violations of the quantum regression theorem, see Eq. (60), can be written as a function of the difference between the central frequencies, $\Delta \omega=\omega_{0,1}-\omega_{0,2}$, as well as of the difference between the corresponding widths, $\Delta \delta \omega=\delta \omega_{1}-\delta \omega_{2}$. If we assume that the two central frequencies are equal, $\omega_{0,1}=\omega_{0,2}=\omega_{0}$, the evolution of the two-level statistical operator is fixed by a time-local master equation as in Eq. (31), with $\epsilon(t)=-\Delta n \omega_{0}$ and

$$
\mathcal{D}(t)=\Delta n \frac{\delta \omega_{1} e^{-\Delta n \delta \omega_{1} t}+r \delta \omega_{2} e^{-\Delta n \delta \omega_{2} t}}{e^{-\Delta n \delta \omega_{1} t}+r e^{-\Delta n \delta \omega_{2} t}} .
$$

The latter is a positive function of time: the reduced dynamics is CP divisible, see Sec. II B, and hence it is Markovian with respect to both the BLP and RHP definitions. Indeed, now we are in the presence of a time-inhomogeneous Markovian dynamics. Nevertheless, as $\gamma\left(t_{2}-t_{1}\right) \neq \gamma\left(t_{2}\right) / \gamma\left(t_{1}\right)$ the quantum regression theorem is violated; see Eq. (60). This is explicitly shown in Fig. 4(a), where $Z$ is plotted as a function of $\Delta \delta \omega=\delta \omega_{1}-\delta \omega_{2}$ and $\Delta n \tau$, with $\tau=t_{2}-t_{1}$. With growing difference between the two widths, as well as the length of the time interval, the deviations from the quantum regression theorem are increasingly strong, up to a saturation value of the estimator $Z$. Contrary to the semigroup case, here, even if the dynamics is Markovian according to both definitions, the actual behavior of the two-time correlation functions cannot be reconstructed by the evolution of the mean values.

Finally, let us consider a frequency distribution as in Eq. (64), but now with $\delta \omega_{1}=\delta \omega_{2}=\delta \omega$ and $\omega_{0,1} \neq \omega_{0,2}$. This frequency distribution has two peaks and the resulting reduced dynamics is non-Markovian [15,46]. In this case the BLP non-Markovianity measure (8) increases with the increasing of the distance between the two peaks, while the estimator $Z$ grows for small values of the distance and then it exhibits an oscillating behavior, see Fig. 4(b). Indeed, for $\Delta \omega=0$ one recovers the semigroup dynamics previously described and, accordingly, $Z$ goes to zero. Summarizing, by varying the distance between the two peaks, one obtains a transition from a Markovian (semigroup) dynamics to a non-Markovian one and, correspondingly, the quantum regression theorem ceases to be satisfied and is even strongly violated. Nevertheless, the qualitative behavior of, respectively, the non-Markovianity of the reduced dynamics and the violation of the quantum regression theorem appear to be different. (a)

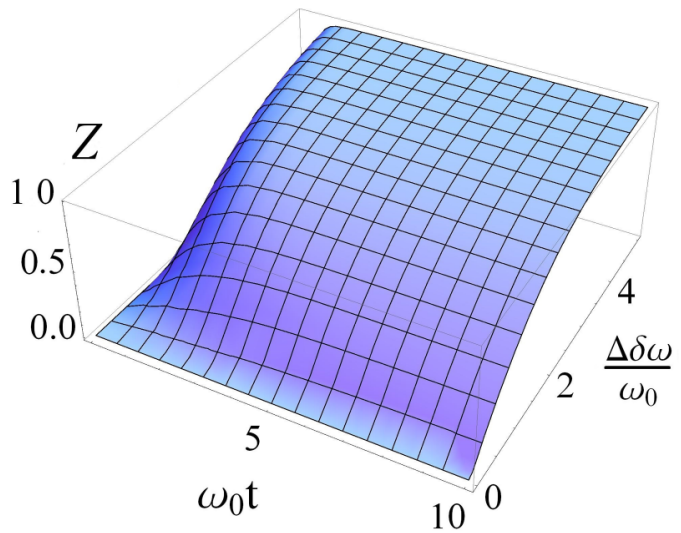

(b)

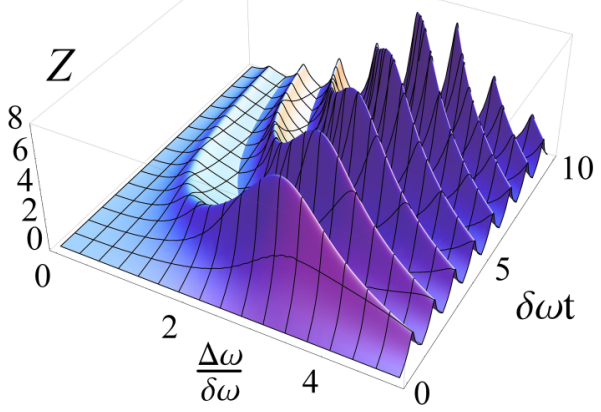

FIG. 4. (Color online) Violation of the quantum regression theorem, as quantified by the estimator $Z$ in Eq. (60) (a) in the timeinhomogeneous Markovian case, $\omega_{0,1}=\omega_{0,2}=\omega_{0}$, as a function of $\Delta \delta \omega=\delta \omega_{1}-\delta \omega_{2}$ and $\omega_{0} \tau=\omega_{0}\left(t_{2}-t_{1}\right)$, for $\omega_{0} t_{1}=1$ and $r=1$; (b) in the non-Markovian case, $\delta \omega_{1}=\delta \omega_{2}=\delta \omega$, as a function of $\Delta \omega_{0}=\omega_{0,1}-\omega_{0,2}$ and $\delta \omega \tau$, for $\delta \omega t_{1}=1$ and $r=2$. In both panels $\Delta n=1$.

\section{CONCLUSIONS}

We have explored the relationship between two criteria for Markovianity of a quantum dynamics, namely the CP divisibility of the quantum dynamical map and the behavior in time of the trace distance between two distinct initial states, and the validity of the quantum regression theorem, which is a statement relating the behavior in time of the mean values and of the two-time correlation functions of system operators. The first open system considered is a two-level system affected by a bosonic environment through a dephasing interaction. For a class of spectral densities with exponential cutoff and power-law behavior at low frequencies we have studied the onset of non-Markovianity as a function of the coupling strength and of the power determining the low-frequency behavior, further giving an exact expression for the corresponding non-Markovianity measures. The deviation from the quantum regression theorem has been estimated evaluating the relative error made in replacing the exact two-time correlation function for the system operators with the expression reconstructed by the evolution of the corresponding mean values. It appears that the validity of the quantum regression theorem represents a stronger requirement than 
Markovianity, according to either criteria, which in this case coincide but quantify non-Markovianity in a different way and exhibit distinct performances in their dependence on strength of the coupling and low-frequency behavior. We have further considered an all-optical realization of a dephasing interaction, as recently exploited for the experimental investigation of non-Markovianity, obtaining also in this case, for different choices of the frequency distribution, significant violations to the quantum regression theorem even in the presence of a Markovian dynamics. This can be understood in terms of the different relevance of the quantum correlations between system and environment in the development of the joint dynamics. While these correlations may not be strong enough to induce a non-Markovian time development of the reduced statistical operator, obtained by directly taking the partial trace, they can still importantly affect the time development of the correlation functions, in which the partial trace is taken only after considering the product of different Heisenberg operators.

These results suggest that indeed the recently introduced new definitions of quantum non-Markovianity provide a weaker requirement with respect to the classical notion of Markovian classical process. In this respect, further and more stringent notions of Markovian quantum dynamics can therefore be introduced, e.g., relying on validity of the quantum regression theorem [17]. Our analysis however also shows that the non-Markovianity of the quantum dynamics, as assessed according to the trace distance criterion, appears to behave differently with respect to violation of the quantum regression theorem in the dependence of relevant model parameters such as the coupling strength in the pure dephasing model. Despite its simplicity, this model suggests that the features of being Markovian and of obeying the quantum regression theorem actually witness different aspects of the quantum dynamics. To better grasp this point further models should be considered and analyzed in detail.

This fact further suggests critically discussing what should be the meaning and relevance of a notion of non-Markovian quantum dynamics. Especially in view of the fact that a simple and relevant hierarchy of non-Markovian quantum processes, analogous to the characterization of classical processes, appears unfeasible due to the intrinsic structure of quantum mechanics, major emphasis should be put on the connection between different indicators and observable properties. In this respect the quantum regression theorem refers to quantities, namely correlation functions, whose physical relevance is well established; think, e.g., of the connection between power spectra and autocorrelation functions. Among the newly introduced signatures of non-Markovian quantum dynamics, the notion of Markovianity based on trace distance, without asking for an explicit exact knowledge of the dynamical equations, allows for a direct experimental check, and has been most recently shown to be able to detect relevant modification in the system-environment dynamics, such as a phase transition [48]. The analysis and comparison of utterly different quantities and concepts which can be related to the notion of memory in a quantum dynamics, such as those considered in the present paper, will help in identifying their possible relevance in unveiling physical interesting phenomena.

\section{ACKNOWLEDGMENTS}

The authors gratefully acknowledge financial support by the EU projects COST Action MP 1006 and NANOQUESTFIT.

\section{APPENDIX: ALTERNATIVE EXPRESSION OF THE DEPHASING FUNCTION}

Starting from Eq. (40), namely

$$
\mathcal{D}_{s}(t)=\frac{\lambda \Omega \Gamma(s)}{\left[1+(\Omega t)^{2}\right]} \sin [s \arctan (\Omega t)],
$$

and exploiting the identities

$$
\sin [\arctan (x)]=\frac{x}{\sqrt{1+x^{2}}}, \quad \cos [\arctan (x)]=\frac{1}{\sqrt{1+x^{2}}}
$$

together with

$$
\sin (s x)=\sum_{k=0}\left(\begin{array}{l}
s \\
k
\end{array}\right)[\cos (x)]^{k}[\sin (x)]^{s-k} \sin \left[\frac{\pi}{2}(s-k)\right],
$$

we can come to the compact expression (41)

$$
\begin{aligned}
\mathcal{D}_{s}(t) & =\frac{\lambda \Omega \Gamma(s)}{2 i\left[1+(\Omega t)^{2}\right]^{s}}\left[\sum_{k=0}\left(\begin{array}{l}
s \\
k
\end{array}\right)(\Omega t)^{s-k}\left[i^{s-k}-(-i)^{s-k}\right]\right] \\
& =\frac{\lambda \Omega \Gamma(s)}{2 i\left[1+(\Omega t)^{2}\right]^{s}}\left[(1+i \Omega t)^{s}-(1-i \Omega t)^{s}\right] \\
& =\lambda \Omega \Gamma(s) \frac{\operatorname{Im}\left[(1+i \Omega t)^{s}\right]}{\left[1+(\Omega t)^{2}\right]^{s}} .
\end{aligned}
$$

[1] G. Lindblad, Commun. Math. Phys. 48, 119 (1976).

[2] G. Lindblad, Commun. Math. Phys. 65, 281 (1979).

[3] M. M. Wolf, J. Eisert, T. S. Cubitt, and J. I. Cirac, Phys. Rev. Lett. 101, 150402 (2008).

[4] H.-P. Breuer, E.-M. Laine, and J. Piilo, Phys. Rev. Lett. 103, 210401 (2009).

[5] H.-P. Breuer, J. Phys. B 45, 154001 (2012).

[6] Á. Rivas, S. F. Huelga, and M. B. Plenio, Phys. Rev. Lett. 105, 050403 (2010).

[7] X.-M. Lu, X. Wang, and C. P. Sun, Phys. Rev. A 82, 042103 (2010).
[8] S. Luo, S. Fu, and H. Song, Phys. Rev. A 86, 044101 (2012).

[9] S. Lorenzo, F. Plastina, and M. Paternostro, Phys. Rev. A 88, 020102(R) (2013).

[10] B. Bylicka, D. Chruściński, and S. Maniscalco, arXiv:1301.2585.

[11] D. Chruściński and S. Maniscalco, Phys. Rev. Lett. 112, 120404 (2014).

[12] F. F. Fanchini, G. Karpat, Çakmak, Castelano, G. H. Aguilar, O. Jiménez Farías, S. P. Walborn, P. H. Souto Ribeiro, and M. C. de Oliveira, Phys. Rev. Lett. 112, 210402 (2014). 
[13] H.-P. Breuer and F. Petruccione, The Theory of Open Quantum Systems (Oxford University Press, Oxford, 2002).

[14] C. W. Gardiner and P. Zoller, Quantum Noise: A Handbook of Markovian and Non-Markovian Quantum Stochastic Methods with Applications to Quantum Optics (Springer, Berlin, 2004).

[15] B.-H. Liu, L. Li, Y.-F. Huang, C.-F. Li, G.-C. Guo, E.-M. Laine, H.-P. Breuer, and J. Piilo, Nat. Phys. 7, 931 (2011).

[16] B. Vacchini, A. Smirne, E.-M. Laine, J. Piilo, and H.-P. Breuer, New J. Phys. 13, 093004 (2011).

[17] N. Lo Gullo, I. Sinayskiy, T. Busch, and F. Petruccione, arXiv:1401.1126.

[18] C. Addis, B. Bylicka, D. Chruściński, and Sabrina Maniscalco, arXiv:1402.4975.

[19] Á. Rivas, S. F. Huelga, and M. B. Plenio, arXiv:1405.0303 [Rep. Prog. Phys. (to be published)].

[20] V. Gorini, A. Kossakowski, and E. C. G. Sudarshan, J. Math. Phys. 17, 821 (1976).

[21] M. Nielsen and I. Chuang, Quantum Computation and Quantum Information (Cambridge University Press, Cambridge, 2000).

[22] S. Wissmann, A. Karlsson, E.-M. Laine, J. Piilo, and H.-P. Breuer, Phys. Rev. A 86, 062108 (2012).

[23] B.-H. Liu, S. Wißmann, X.-M. Hu, C. Zhang, Y.-F. Huang, C.-F. Li, G.-C. Guo, A. Karlsson, J. Piilo, and H.-P. Breuer, arXiv:1403.4261.

[24] J.-S. Tang, C.-F. Li, Y.-L. Li, X.-B. Zou, G.-C. Guo, E.-M. Laine, H.-P. Breuer, and J. Piilo, Europhys. Lett. 97, 10002 (2012).

[25] B. H. Liu, D.-Y. Cao, Y.-F. Huang, C.-F. Li, G.-C. Guo, H.-P. Breuer, E.-M. Laine, and J. Piilo, Sci. Rep. 3, 1781 (2013).

[26] E.-M. Laine, J. Piilo, and H.-P. Breuer, Phys. Rev. A 81, 062115 (2010).

[27] A. Rivas and S. F. Huelga, Open Quantum Systems: An Introduction, Springer Briefs in Physics (Springer, 2012).

[28] M. D. Choi, Lin. Alg. Appl. 10, 285 (1975).
[29] P. Haikka, J. D. Cresser, and S. Maniscalco, Phys. Rev. A 83, 012112 (2011).

[30] D. Chruściński, A. Kossakowski, and Á. Rivas, Phys. Rev. A 83, 052128 (2011).

[31] H. Carmichael, An Open Systems Approach to Quantum Optics (Springer-Verlag, Berlin, 1993).

[32] S. Swain, J. Phys. A: Math. Gen. 14, 2577 (1981).

[33] R. Dümcke, J. Math. Phys. 24, 311 (1983).

[34] E. B. Davies, Commun. Math. Phys. 39, 91 (1974); Math. Ann. 219, 147 (1976).

[35] V. Gorini and A. Kossakowski, J. Math. Phys. 17, 1298 (1976); A. Frigerio and V. Gorini, ibid. 17, 2123 (1976).

[36] P. Talkner, Ann. Phys. 167, 390 (1986).

[37] G. W. Ford and R. F. O’Connell, Phys. Rev. Lett. 77, 798 (1996).

[38] M. Lax, Phys. Rev 172, 350 (1968).

[39] W. G. Unruh, Phys. Rev. A 51, 992 (1995).

[40] A. Ferraro, S. Olivares, and M. G. A. Paris, Gaussian States in Quantum Information (Bibliopolis, Naples, 2005).

[41] H.-S. Zeng, N. Tang, Y.-P. Zheng, and G.-Y. Wang, Phys. Rev. A 84, 032118 (2011).

[42] P. Haikka, T. H. Johnson, and S. Maniscalco, Phys. Rev. A 87, 010103(R) (2013).

[43] H.-S. Goan, C.-C. Jian, and P.-W. Chen, Phys. Rev. A 82, 012111 (2010).

[44] E.-M. Laine, J. Piilo, and H.-P. Breuer, Europhys. Lett. 92, 60010 (2010).

[45] L. Mazzola, C. A. Rodríguez-Rosario, K. Modi, and M. Paternostro, Phys. Rev. A 86, 010102(R) (2012).

[46] A. Smirne, L. Mazzola, M. Paternostro, and B. Vacchini, Phys. Rev. A 87, 052129 (2013).

[47] Á. Rivas, A. D. Plato, S. F. Huelga, and M. B. Plenio, New J. Phys. 12, 113032 (2010).

[48] M. Gessner, M. Ramm, H. Haeffner, A. Buchleitner, and H.-P. Breuer, arXiv:1403.4066. 